\title{
SLAC's polarized electron source laser system for the E-158 parity violation experiment
}

Axel Brachmann, Ray K. Alley, M. J. Browne, G. D. Cates, James Clendenin, et al.

Axel Brachmann, Ray K. Alley, M. J. Browne, G. D. Cates, James Clendenin, J. deLamare, Josef C. Frisch, T. Galetto, E. W. Hughes, Thomas Brian Humensky, K. S. Kumar, Peter A. Mastromarino, J. Sodja, James L. Turner, Klaus $\mathrm{H}$. Witte, Michael B. Woods, "SLAC's polarized electron source laser system for the E-158 parity violation experiment," Proc. SPIE 4632, Laser and Beam Control Technologies, (4 June 2002); doi: 10.1117/12.469769

Event: High-Power Lasers and Applications, 2002, San Jose, California, United States 


\title{
SLAC's polarized electron source laser system for the E-158 parity violation experiment
}

\author{
A. Brachmann ${ }^{a}$, R. Alley ${ }^{b}$, M. J. Browne ${ }^{a}$, G. D. Cates $^{c}$, J. Clendenin ${ }^{a}$, J. deLamare $^{a}$, \\ J. Frisch ${ }^{a}$, T. Galetto ${ }^{a}$, E. W. Hughes ${ }^{d}$, T. B. Humensky ${ }^{e}$, K. S. Kumar ${ }^{f}$, P. Mastromarino ${ }^{d}$, \\ J. Sodja ${ }^{a}$, J. Turner ${ }^{a}$, K. Witte ${ }^{g}$, and M. Woods ${ }^{a}$ \\ ${ }^{a}$ Stanford Linear Accelerator Center, Menlo Park, CA \\ ${ }^{b}$ Illumina, Inc., San Diego, CA \\ ${ }^{c}$ University of Virginia, Charlottesville, VA \\ ${ }^{d}$ California Institute of Technology, Pasadena, CA \\ ${ }^{e}$ Princeton University, Princeton, NJ \\ fUniversity of Massachusetts, Amherst, MA \\ ${ }^{g}$ Resonant Optics Corporation, San Martin, CA
}

\begin{abstract}
SLAC E158 is an experiment to make the first measurement of parity violation in Møller scattering. The left-right cross-section asymmetry in the elastic scattering of a $45-\mathrm{GeV}$ polarized electron beam off unpolarized electrons in a liquid hydrogen target will be measured to an accuracy of better than $10^{-8}$, with the expected Standard Model asymmetry being approximately $10^{-7}$. An intense circularly polarized laser beam for the polarized electron source is required with the ability to quickly switch between left and right polarization states with minimal left-right asymmetries in the parameters of the electron beam. This laser beam is produced by a unique SLAC-designed, flash-lamp pumped, Ti:Sapphire laser. We present this laser system design and initial results from recent commissioning runs.
\end{abstract}

Keywords: Parity violation, polarized electrons, Ti:sapphire laser

\section{INTRODUCTION}

The Stanford Linear Accelerator Center (SLAC) has a distinguished history of providing polarized electron beams for use in high energy physics experiments, including important tests of the Standard Model of particle physics and detailed studies of the spin structure of nucleons. ${ }^{1,2,3}$ SLAC's polarized electron source is based on photoemission from a strained GaAs cathode held at a bias voltage of $-120 \mathrm{kV}$ and pumped by an intense, circularly polarized laser beam. ${ }^{4}$ Two laser systems exist to pump the cathode: an YLF:Ti system that generates short (2 ns) pulses of electrons for SLAC's Positron Electron Project (PEP) rings, and a flash-lamp pumped Ti:Sapphire (the "Flash:Ti") laser used to generate $370 \mathrm{~ns}$ pulses for use in fixed target experiments at SLAC's "End Station A" such as E158.

E158 will make the first measurement of parity violation in Møller scattering, by measuring the asymmetry in the cross section for elastic scattering of longitudinally polarized electrons with an energy of $45 \mathrm{GeV}$ off of an unpolarized electron target:

$$
A_{L R}=\frac{\sigma_{R}-\sigma_{L}}{\sigma_{R}+\sigma_{L}}
$$

where $\sigma_{R}\left(\sigma_{L}\right)$ is the cross-section for incident right- (left-) handed electrons. The experiment will be a stringent test of the Standard Model of particle physics and will be sensitive to new physics beyond the Standard Model. The asymmetry will be measured to an accuracy of better than $10^{-8}$, with the expected Standard Model asymmetry being approximately $10^{-7}$. To achieve this measurement in a reasonable running time, the Flash:Ti system will be used to generate electron pulses containing up to $6 \cdot 10^{11}$ electrons in $370 \mathrm{~ns}$ at a repetition rate of $120 \mathrm{~Hz}$. Table 1 summarizes the parameters for the Flash:Ti laser system. Recent work, described below, has both increased the pulse energy and decreased the energy jitter significantly. Helicity correlations in the 
parameters of the electron beam when it is incident on the E158 target must be held to very small levels to prevent them from introducing systematic errors in the measurement. Averaged over the length of the physics run, the intensity asymmetry $A_{I}$, the energy asymmetry $A_{E}$, and the position difference $\mathrm{D}_{X(Y)}$ must be held below the following limits:

$$
A_{I}=\left\langle\frac{I_{R}-I_{L}}{I_{R}+I_{L}}\right\rangle<2 \cdot 10^{-7}, A_{E}=\left\langle\frac{E_{R}-E_{L}}{E_{R}+E_{L}}\right\rangle<2 \cdot 10^{-8}, D_{X}=\left\langle x_{R}-x_{L}\right\rangle<10 \mathrm{~nm} .
$$

Highly circularly polarized laser light, as well as the ability to control the amount and orientation of residual linear polarization, is necessary to achieve these goals. A pair of Pockels cells sets the beam polarization and allows switching between helicity states on a pulse-by-pulse basis. However, control of the laser polarization is not sufficient. The Pockels cells can steer the beam in a helicity-correlated way, so care must be taken to image the beam from the Pockels cells to the cathode. Active feedbacks on the intensity asymmetry and position differences are also required to ensure that the integrated helicity-correlated systematics converge to zero. Finally, means of introducing slow reversals (i.e., over many-hour timescales rather than pulse-by-pulse) of either the sign of the physics asymmetry or the sign of certain classes of systematics are useful in gaining additional cancellations of systematic errors. All of these features have been implemented on the Flash:Ti system for E158 and are described below. This paper will focus on the Flash:Ti laser system for the polarized

Table 1. Flash:Ti laser parameters. The last three rows represent measurements on the electron beam. The last two rows represent results achieved using active feedbacks during a recent commissioning run. ${ }^{5}$

\begin{tabular}{|l|l|}
\hline Wavelength range & $750-850 \mathrm{~nm}$ \\
\hline Repetition rate & $60-120 \mathrm{~Hz}$ \\
\hline Pulse length & $130-370 \mathrm{~ns}$ \\
\hline Pulse energy & $175 \mu \mathrm{J}-1 \mathrm{~mJ}$ \\
\hline Energy jitter & $0.5 \%-1.5 \%$ \\
\hline Position jitter at photocathode & $<100 \mu \mathrm{m}$ \\
\hline Helicity-correlated intensity asymmetry & $2 \cdot 10^{-7}$ \\
\hline Helicity-correlated position difference & $20 \mathrm{~nm}$ \\
\hline
\end{tabular}

source. It was originally designed and commissioned in 1993 and has seen extensive upgrades in preparation for E158. Earlier descriptions of the laser system are given by Witte ${ }^{6}$ and Alley et al. ${ }^{4}$ A schematic of the polarized source as it is configured for E158 is shown in Fig. 1. The laser system is housed in an environmentally controlled room outside of the accelerator tunnel. The laser system naturally breaks into a set of subsystems that correspond to several optical benches. The "Flash:Ti Bench" holds the laser cavity and pulse-shaping optics. The "Diagnostics Bench" has photodiodes for monitoring the laser's intensity and temporal profile and a spectrometer. The "Helicity Control Bench" houses the optics for controlling the polarization state of the beam and for suppressing helicity-correlated systematics. A 20 m-long Optical Transport System (OTS) carries the beam into the accelerator tunnel, where it crosses the "Cathode Diagnostics Bench" and is directed onto the cathode of the polarized gun. The resulting photoelectrons are bent through $38^{\circ}$ and enter the accelerator. This paper is organized in a series of sections that describe each optical subsystem and its performance. Frequent reference is given to results from a test run, T-437, ${ }^{5}$ which took place in November 2000 and commissioned the polarized source for E158 and to a set of E158 engineering runs that took place from January-May 2001 and provided significant long-term experience with the laser system. E158 is scheduled to have a physics run during the spring of 2002 . 


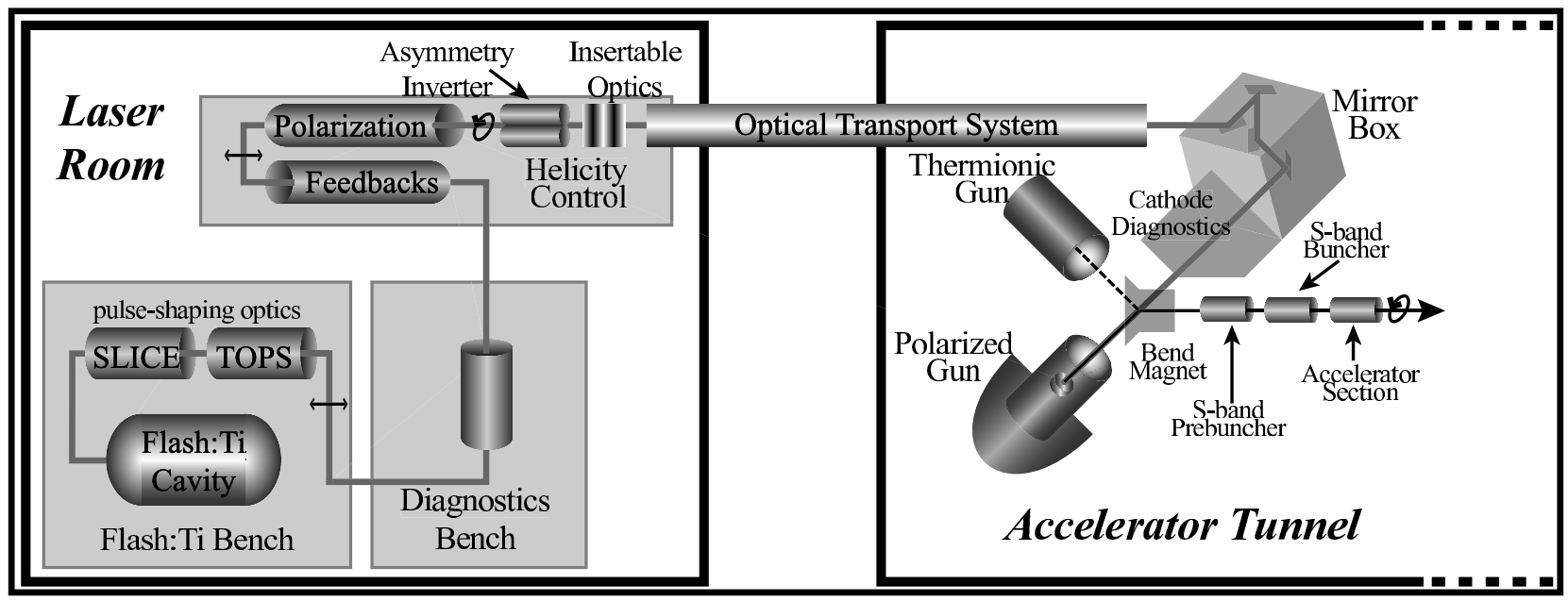

Figure 1. An overview schematic of the Polarized Electron Source as it is configured for E158. A flashlamp-pumped Ti:Sapphire laser provides a $370 \mathrm{~ns}, 1 \mathrm{~mJ}$ pulse that is circularly polarized and transported into the accelerator tunnel, where it strikes the cathode of the polarized electron gun. Photoelectrons are accelerated away from the cathode by a $120 \mathrm{kV}$ potential and enter the accelerator.

\section{FLASH:TI BENCH}

\subsection{Pump Chamber and Laser Cavity}

The Flash:Ti pump chamber was designed at SLAC and built by Big Sky Laser Technologies. ${ }^{7}$ The rod-shaped Ti:Sapphire crystal is pumped by two flashlamps, each of which is associated with an elliptically shaped reflector. The original commercial silver coatings of the reflectors and pump chamber end plates have been replaced by rhodium. This substantially increased their mechanical and chemical surface durability and eliminated the need to purge the pump chamber with nitrogen. The pump chamber parts can be exposed to air while they are handled without the risk of corrosion. Two cylindrical flashlamps are used for this system (model L8061E, T J Sales Associates Inc, ILC ${ }^{8}$ ). They have the following specifications: ID $4.8 \mathrm{~mm}$, OD $5.98 \mathrm{~mm}, 7.6^{\prime \prime}$ arc length, Ce-doped quartz walls, and 450 Torr Xe filling. The output of the flashlamps is focused on the center of a 4 mm-diameter 0.1\% doped Ti:Sapphire laser rod of 6.4 inch length (Union Carbide Crystal Products ${ }^{9}$ ). The end surfaces of the laser rod are cut perpendicular to the c-axis of the crystal and are antireflection coated at 850 nm. The rod, flashlamps, and pump chamber are cooled by a closed loop of ultra-pure water. The rod flow tube surrounds the laser rod and its material (KTF-2 fluorescent converter, Kigre, Inc. ${ }^{10}$ ) acts as a UV filter to prevent excessive solarization of the Ti:Sapphire material. A $90 \%$ reflectivity planar output coupler and a $99.9 \%$ reflectivity end mirror with a 5-meter concave curvature form the laser cavity. Both mirrors have narrow band coatings centered at $850 \mathrm{~nm}$. A single quartz plate of $2 \mathrm{~mm}$ thickness acts as the wavelength selecting element. The quartz plate is mounted to allow for horizontal and vertical rotation. In the horizontal plane the plate is set to the Brewster angle of $\sim 57$ degrees. The effective refractive index $\mathrm{n}_{e}$ of the quartz plate depends on the angle between the electric field vector and the optical axis of the quartz plate. By rotation in the vertical plane birefringent wavelength tuning is achieved and the transmission is optimized for the desired output wavelength of $852 \mathrm{~nm}$ with a bandwidth of $\sim 0.7 \mathrm{~nm}$ (FWHM). An additional half-wave plate compensates for the arbitrary rotation of the Ti:Sapphire laser rod and thereby guarantees that s-polarization is incident on the first 45-degree mirror outside the cavity. A scheme of the laser cavity is depicted in Fig. 2.

\subsection{Flow System}

The cooling water flow is a closed system and can be refilled by on-site low conductivity water. The scheme utilized is given in Fig. 3. The system is designed to ensure a constant temperature of $76{ }^{\circ} \mathrm{F}$ and inert water conditions. The main loop operates at a flow rate of $2.5 \mathrm{GPM}$. Ultra pure water quality $(>15 \mathrm{M} \Omega$ ) is established by a 1 GPM polishing loop which contains a $0.2 \mu \mathrm{m}$ particle filter, a deionization filter, an organic filter, and an 


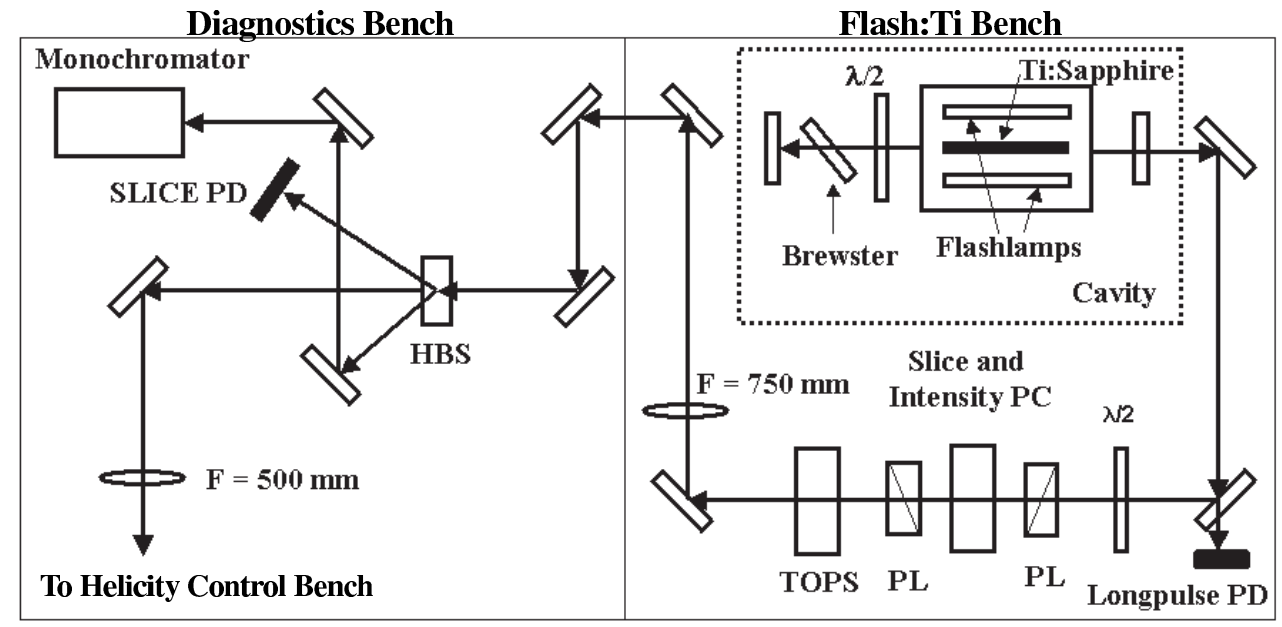

Figure 2. Scheme of optical layout and laser cavity $(\lambda / 2$ : half-wave plate, PD: photo diode, PL: polarizer, PC: Pockels cell, HBS: harmonic beam sampler).

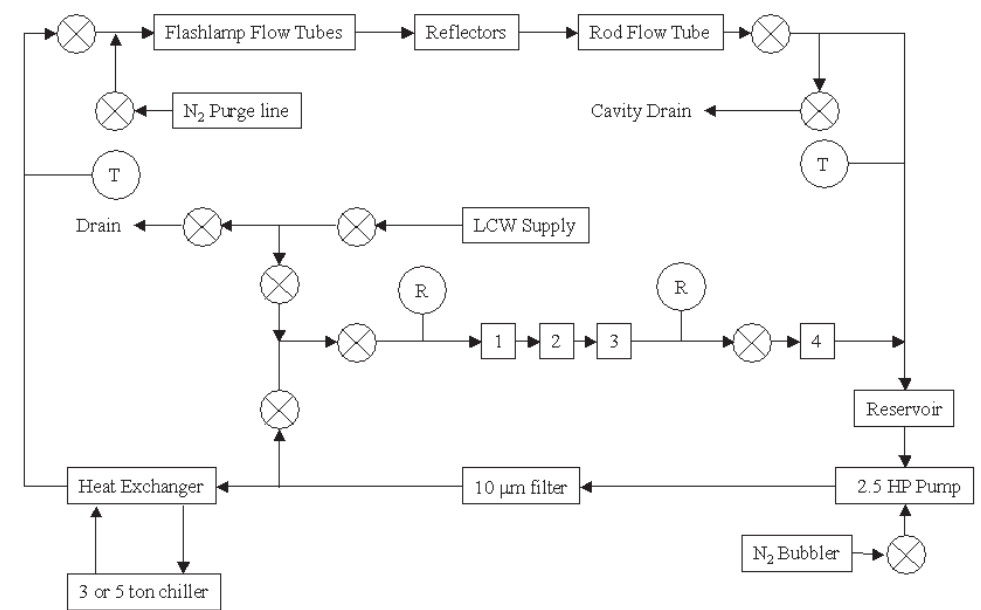

Figure 3. Cooling water system components and water flow, including resistivity sensor ((R)), temperature sensor ((I)), valve $(\bigotimes)$, on-site low conductivity water (LCW), oxygen removal cartridge (1), activated carbon - organics filter (2), mixed bed deionizer (3), and submicron filter (4).

oxygen filter. A nitrogen bubbler removes larger amounts of dissolved oxygen from the reservoir. This was of particular importance when the silver-coated reflectors were in use. The cooling water constantly flows through a $10 \mu \mathrm{m}$ particle filter and a heat exchanger. Either a 3-ton or 5-ton chiller can be used to provide the cooling for the heat exchanger. Interlocks are connected to water resistivity and temperature sensors which shut off the laser power supply if the values are out of tolerance. For flashlamp changes the laser head can be drained and purged by a separate $\mathrm{N}_{2}$ supply.

\subsection{Modulator}

The modulator was designed and built by SLAC personnel. A $1.2 \mu \mathrm{F}$ capacitor is charged by a $10 \mathrm{kV}, 8 \mathrm{~kJ} / \mathrm{s}$ power supply. Upon ignition of a thyratron, the capacitor is discharged through the two flashlamps in series. This produces an over-damped electrical pulse where the pulse's characteristics are set by the capacitance of the capacitor and the stray inductance and resistance of the circuit components. The pulse has a peak current of $1 \mathrm{kA}$ and a pulse duration of $22 \mu \mathrm{s}$. Between pulses, a current through the flashlamps is maintained by a simmer power supply in parallel. The simmer current reduces the high voltage needed for conduction in the 


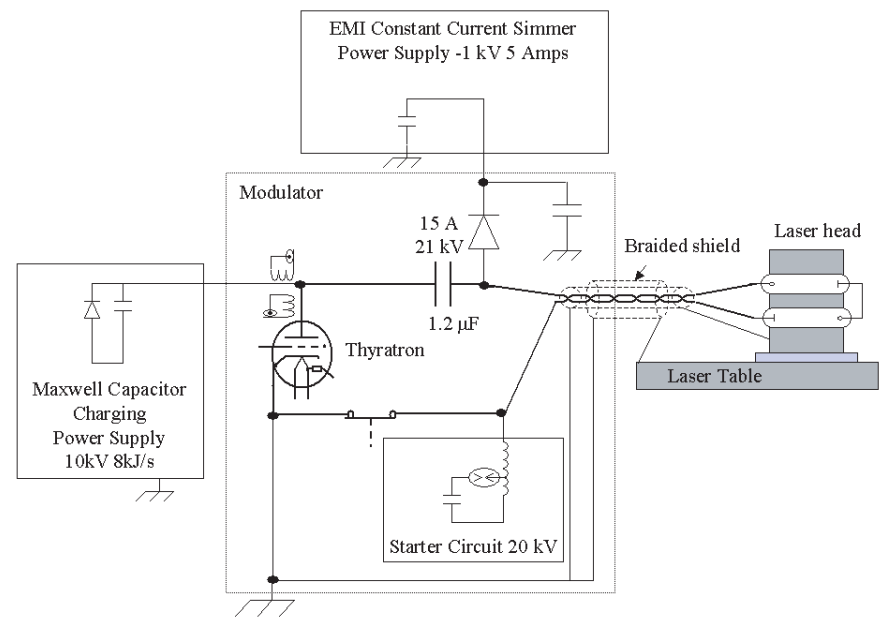

Figure 4: Schematic circuit diagram of laser power supply.

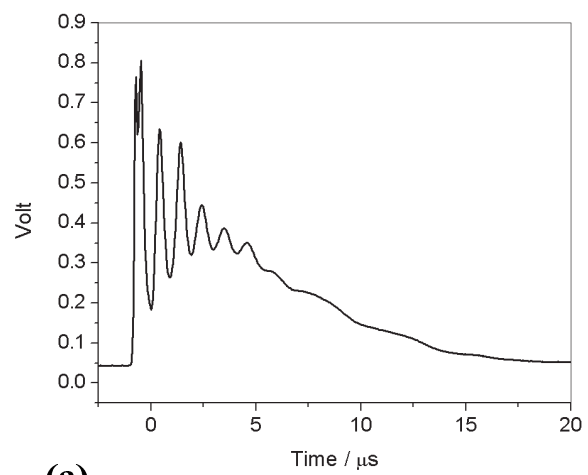

(a)

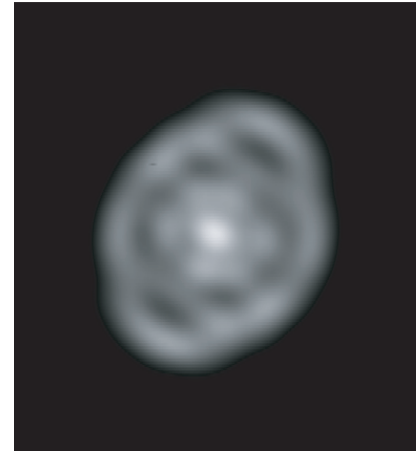

(b)

Figure 5: (a) Photodiode signal of Ti:Sapphire laser pulse. (b) Ti:Sapphire laser beam spatial profile.

lamps and thereby extends their lifetime. The modulator and the laser cavity were designed to operate at 120 pps. The recent test run T-437 and the E158 engineering run were conducted at a repetition rate of 60 pps. The schematic circuit of the power supply is shown in Fig. 4.

\subsection{Laser Performance and Pulse Shaping}

The typical laser output power with the cavity tuned to $852 \mathrm{~nm}$ was $\sim 15$ to $20 \mathrm{~mJ}$ in a $10 \mu$ s long pulse. The temporal structure of the pulse is depicted in Fig. 5a. For the E158 engineering run a 130 ns long pulse (FWHM) was sliced out the laser pulse at the point of lowest intensity jitter. This was achieved using a pair of crossed polarizers and a Pockels cell, which is driven by a commercial high voltage pulser (Directed Energy Inc. ${ }^{11}$ ). This Pockels cell is also used for pulse intensity control. Up to $175 \mu \mathrm{J} /$ pulse were produced in the modified pulse with a $1.5 \% \mathrm{rms}$ pulse-to-pulse intensity jitter. To provide the necessary pulse shape the laser must be operated multi-modal. A typical laser profile is presented in Fig. 5b. To minimize the shot-to-shot energy jitter, following the E158 engineering run the laser was operated with different end mirror combinations and variations of high voltage and thyratron reservoir settings. The best results were achieved with the above described end mirrors. Combined with the wavelength change to $805 \mathrm{~nm}$, these end mirrors yielded an energy of $1 \mathrm{~mJ}$ in $370 \mathrm{~ns}$ and an energy jitter of $0.5 \%$. The long-term stability of these results is now under study.

Aging of flashlamps and thyratron required small adjustments of high voltage and thyratron reservoir throughout the period of the experiment. Slow drifts in laser power and stability caused by humidity and 


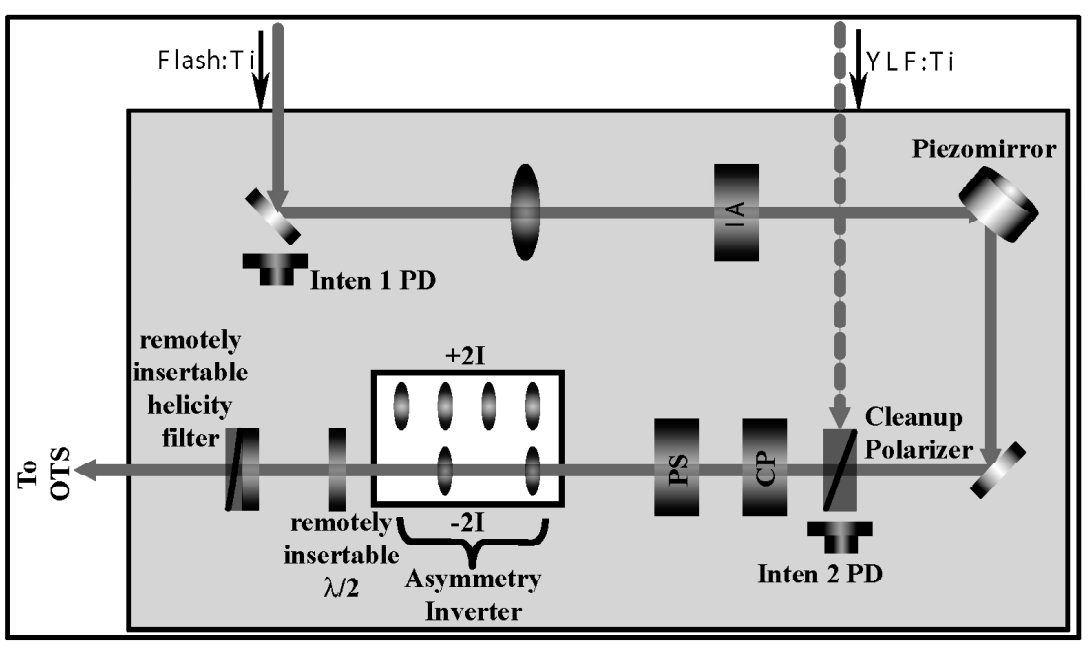

Figure 6. The Helicity Control Bench. This bench contains the optics for control of the laser beam's polarization and helicity-correlated systematics.

temperature were minimized by appropriate air conditioning and humidity control. The temperature and humidity in the laser room were stable at $23 \pm 0.5^{\circ} \mathrm{C}$ and $36 \pm 1 \%$, respectively.

The beam divergence is dominated by thermal lensing of the flashlamp-pumped Ti:Sapphire rod with a corresponding focal length of $\sim 1.2$ meter. The diverging beam is collimated by additional lenses located downstream of the pulse-shaping optics. Fig. 2 shows a scheme of the optical layout containing the described pulse shaping and steering optics. All steering mirrors are $45^{\circ}$ dielectric mirrors.

The maintenance of the laser system during the E158 engineering runs ( 5 months continuous operation at $60 \mathrm{~Hz}$ ) consisted of flashlamp changes every 28 days and cooling water system filter changes after a period of 6 months. No significant drop in laser performance was observed due to the aging of flashlamps or water filters.

\section{DIAGNOSTICS}

The laser performance is monitored by photodiodes. Either leakage light through $45^{\circ}$ folding mirrors or a sampled beam (1\%) provides a sufficient signal for routine intensity, jitter and wavelength measurements. One photodiode installed upstream of the pulse-shaping Pockels cell / polarizer optics monitors the Ti:Sapphire laser output (Longpulse PD). Downstream of the pulse-shaping optics two one-percent fractions of the laser beam are separated by a harmonic beam sampler (Gentec Electro-Optics ${ }^{12}$ ). One fraction is used to monitor the intensity of the sliced pulse (SLICE PD). The second fraction is focused onto a scanning monochromator for wavelength measurements. The location of photodiodes used for diagnostics is given in Fig. 2.

\section{HELICITY CONTROL}

The Helicity Control Bench optics determine the laser beam's polarization and control its helicity-correlated systematics. Helicity correlations in electron beam parameters (intensity, energy, position, and size) can in general be traced back to helicity correlations in laser beam parameters. The Helicity Control Bench optics (shown below in Fig. 6) were designed to generate highly circularly polarized light of either helicity while minimizing the helicity correlations introduced, as well as to provide active means of controlling helicity correlations. The optics on the Helicity Control Bench fall into two subsets: those used primarily to control the laser beam polarization and those used solely to control helicity-correlated systematics.

\subsection{Polarization and Systematics Control Optics}

A linear polarizer (the "Cleanup Polarizer") and a pair of Pockels cells (the "Circular Polarization," or CP, and the "Phase Shift," or PS, cells) determine the polarization of the beam. The Cleanup Polarizer is used to produce horizontally polarized light free of any ellipticity that may have crept into the polarization since the 
pulse-shaping optics. The Cleanup Polarizer also combines the YLF:Ti beam used to generate electrons for the $\mathrm{PEP}$ rings with the Flash:Ti. The $\mathrm{CP}$ cell acts as a quarter-wave plate with its fast axis at $45^{\circ}$ from the vertical whose retardation flips sign pseudorandomly ${ }^{13}$ on a pulse-by-pulse basis, generating circularly polarized light of either helicity. Its quarter-wave voltage is approximately $2.8 \mathrm{kV}$. The PS cell, with a vertical fast axis, is pulsed at a low voltage and is used to compensate for residual linear polarization along the axes at $\pm 45^{\circ}$ that arises from either residual birefringence in the Pockels cell or phase shifts in the optics between the Pockels cells and the photocathode. The Pockels cell mounts are adjustable in pitch, yaw, and roll to allow for proper alignment of the Pockels cells' optic axes with the laser beam and their fast axes with the laser beam's polarization.

It is useful to identify the particular phases in the optics system that lead to helicity-correlated beam parameters. Toward this end, we will develop an expression for the helicity-correlated intensity asymmetry of light that is incident on the photocathode. The electric field of a polarized laser beam in its most general form is

$$
\vec{E}=E_{0}\left(\cos \theta \cdot \hat{x}+e^{i \phi} \sin \theta \cdot \hat{y}\right) \cdot e^{i(k z-\omega t)}=\left[\begin{array}{c}
\cos \theta \\
e^{i \phi} \sin \theta
\end{array}\right],
$$

where the vector expression is the Jones matrix notation. The polarization of the beam can be understood in terms of its Stokes parameters:

$$
\begin{gathered}
S_{1}=\cos 2 \theta=\frac{X-Y}{X+Y}, \\
S_{2}=-\sin 2 \theta \cos \phi=\frac{U-V}{U+V}, \\
S_{3}=\sin 2 \theta \sin \phi=\frac{R-L}{R+L}, \\
\left(S_{1}^{2}+S_{1}^{2}\right)+S_{3}^{2}=L^{2}+C^{2}=1,
\end{gathered}
$$

where $S_{1}$ is a measure of linear polarization along the horizontal and vertical axes, $S_{2}$ is a measure of linear polarization along the axes at $\pm 45^{\circ}$ to the vertical, $S_{3}$ is a measure of the degree of circular polarization, $\mathrm{X}$ and $\mathrm{Y}$ represent intensities along horizontal and vertical axes, $\mathrm{U}$ and $\mathrm{V}$ represent intensities along axes at $\pm 45^{\circ}$ to the vertical, and $\mathrm{R}$ and $\mathrm{L}$ represent intensities for right- and left-circularly polarized light. Because the linear and circular polarizations ( $\mathrm{L}$ and $\mathrm{C}$, respectively) add in quadrature, a small imperfection in the circular polarization will give rise to a large contribution of linear polarization. The phase shifts induced by the $\mathrm{CP}$ and PS cells are proportional to the voltage applied to each cell:

$$
\delta_{C P}=\frac{V_{C P}}{V_{\lambda / 4}} \frac{\pi}{2}, \delta_{P S}=\frac{V_{P S}}{V_{\lambda / 4}} \frac{\pi}{2},
$$

where $V_{\lambda / 4}$ is the voltage required for quarter-wave retardation for each Pockels cell. Starting with a Jones vector of $\left[\begin{array}{l}1 \\ 0\end{array}\right]$ for horizontally polarized light after the Cleanup Polarizer, we find that following the PS cell the electric vector takes the form

$$
\left[\begin{array}{c}
\cos \delta_{C P} / 2 \\
e^{i\left(\frac{\pi}{2}-\delta_{P S}\right)} \sin \delta_{C P} / 2
\end{array}\right] \text {. }
$$

Making the appropriate choices of $\delta_{C P}$ and $\delta_{P S}$ will allow the generation of arbitrary elliptically polarized light. In particular, we note that the Stokes parameter $S_{1}$ is sensitive to $\delta_{C P}$, while $S_{2}$ is sensitive to $\delta_{P S}$. Ideally, to achieve perfect circular polarization one would simply choose $V_{C P}= \pm V_{\lambda / 4}$ and $V_{P S}=0$ for the two helicity states. Unfortunately, imperfections in the alignment of the Pockels cells' axes with respect to the incoming linear polarization, residual birefringence within the Pockels cells, and birefringence in the optics between the Pockels cells and the photocathode complicate the situation. A convenient formalism for describing imperfections in the laser beam's circular polarization is to assume that the CP and PS cells induce retardations of the form

$$
\begin{gathered}
\delta_{C P}^{ \pm}= \pm\left(\frac{\pi}{2}+\alpha_{1}\right)-\Delta_{1} \\
\delta_{P S}^{ \pm}= \pm \alpha_{2}-\Delta_{2}
\end{gathered}
$$



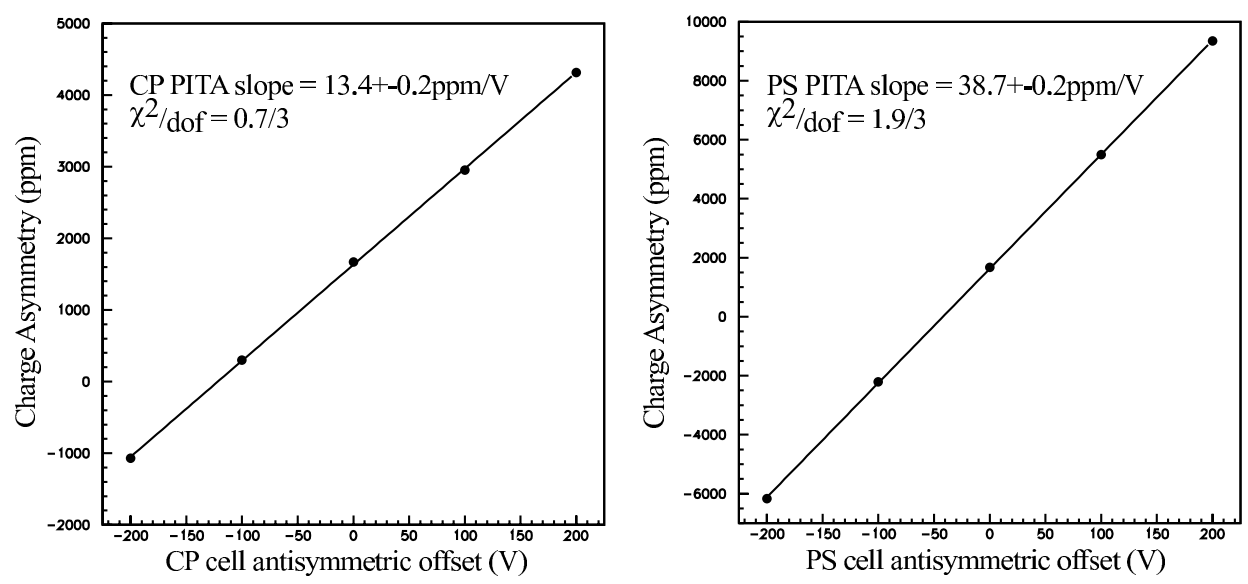

Figure 7. PITA slopes for both the CP and PS cells. Slopes are plotted in terms of ppm of intensity asymmetry per volt of antisymmetric offset voltage applied to the Pockels cells and are typical values for strained GaAs cathodes.

where the superscripts \pm indicate the two helicities and the imperfect phase shifts have been parameterized in terms of symmetric $(\alpha)$ and antisymmetric $(\Delta)$ pieces. We give the phases from the CP cell a subscript "1" because $\delta_{C P}$ is sensitive to Stokes parameter $S_{1}$. Similarly, the phases induced by the PS cell carry a subscript "2" to emphasize that $\delta_{P S}$ is sensitive to Stokes parameter $S_{2}$. Birefringence in the downstream optics will contribute additional antisymmetric phase shifts. It can be shown that when such a laser beam is transmitted through an element with a preferred axis of transmission (an analyzing power), an intensity asymmetry will result that depends solely on the antisymmetric phase shifts (using small angle approximations):

$$
A_{I}=\frac{I^{+}-I^{-}}{I^{+}+I^{-}}=\frac{\epsilon}{T}\left[\left(\Delta_{1}-\Delta_{1}^{0}\right) \cos 2 \theta-\left(\Delta_{2}-\Delta_{2}^{0}\right) \sin 2 \theta\right],
$$

where the ratio $\frac{\epsilon}{T}$ is the strength of the analyzing power, $\theta$ is the angle between the cathode's preferred axis and the horizontal axis, and $\Delta_{1}^{0}$ and $\Delta_{2}^{0}$ represent the phase shifts picked up due to residual birefringence in the Pockels cells and the optical elements downstream of them. We refer to this dependence of charge asymmetry on phase shifts as the Polarization Induced Transport Asymmetry (PITA) effect. ${ }^{14}$ Eqn. 8 remains valid if transmission through an optical element with an analyzing power is replaced by photoemission from a cathode with a linear-polarization-dependent quantum efficiency. The strained GaAs photocathodes in use at SLAC typically have an analyzing power on the order of $0.1 .{ }^{15}$ By adjusting the voltages on the CP and PS cells in an antisymmetric fashion, one can adjust the size of either the Stokes 1 or the Stokes 2 components, and thus the size of the intensity asymmetry. Fig. 7 shows "PITA slopes" for each Pockels cell from the recent test run, T-437. Because there are two free parameters, $\Delta_{1}$ and $\Delta_{2}$, eq. 8 can be used to define a line in "voltage space" along which the intensity asymmetry is zero. We have found empirically that helicity-correlated electron beam position differences correlate with the choice of voltages along this line, making the choice of voltages a useful tool for optimizing position differences. In addition, by moving perpendicular to that line one can correct drifts in phase shifts to keep the intensity asymmetry small. In the future, we will implement a "double feedback" that will use this feature to keep small the corrections induced by the intensity asymmetry feedback that will be discussed below.

The Pockels cells have a residual birefringence that contributes to $\Delta_{1}^{0}$ and $\Delta_{2}^{0}$ and may also depend on position across the face of the crystal. That position-dependent birefringence, coupled to the finite width of the laser beam, can cause the retardation across the laser beam to become position-dependent. This positiondependence gives rise to a position-dependent intensity asymmetry across the profile of the electron beam and can cause the centroid and the rms width of the electron beam to become helicity-correlated. We studied the residual birefringence in six $20 \mathrm{~mm}$ diameter Pockels cells (Cleveland Crystals QX2035 ${ }^{16}$ ) and chose the two with the smallest gradients in birefringence. We placed them on two-axis translation stages in order to optimize in situ the point on the crystals through which the laser beam passes. In addition, we chose to bring 
the beam through a gentle focus at the CP and PS cells. By minimizing the beam diameter, we reduce our sensitivity to the gradient in birefringence across the Pockels cells. However, because the Pockels cells are naturally birefringent (with the optic axis having a significantly different index of refraction than the plane perpendicular to it), it is important to keep the beam well collimated passing through them so that all rays see an equal optical path length. We found the optimum beam sigma to be approximately $1 \mathrm{~mm}$ at the CP cell.

The CP and PS voltages are set initially by inserting an "Helicity Filter" (HF) downstream of them and scanning their voltages. The Helicity Filter is made of a quarter-wave plate and a linear polarizer separated by an air gap such that one circular polarization is transmitted and the other extinguished. Optimizing the CP and PS cell voltages for transmission in one helicity and extinction in the other yields an extinction ratio of $\geq 1000: 1$, implying a circular polarization of $\geq 99.8 \%$ and an unpolarized component of $\leq 0.2 \%$.

When the beam is brought through the remaining optics to the photocathode, birefringence in the optics will induce antisymmetric phase shifts (i.e., $\Delta_{1}$ and $\Delta_{2}$ ), leading to a sizeable intensity asymmetry on the electron beam (typically 0.1-0.5\%). By measuring the PITA slopes for both cells and choosing appropriate voltage offsets, it is easy to reduce the intensity asymmetry below $10^{-4}$. However, since there are two voltages available, it is not obvious which choice of offsets will yield maximal circular polarization on the cathode. One method to determine the best choice of offsets is to measure the electron beam polarization (which will have a $\cos (\phi)$ behavior) for several combinations of voltages that yield a small intensity asymmetry and to identify the peak. Alternatively, one can choose to relax the requirement for maximal circular polarization, and, as discussed above, use the freedom in choice of voltage to minimize position systematics.

\subsection{Active Control and Cancellation of Helicity-Correlated Systematics}

The polarization optimization procedures discussed in the preceding section are not sufficient to meet E158's stringent requirements on helicity-correlated systematics. Active feedback on both the intensity asymmetry and position differences is required. Additional means of generating slow reversals of systematics in order to arrange cancellations prove useful as well, as does imaging the CP and PS cells onto the cathode. Each of these strategies will be described in this section.

The feedback loops used to minimize $\mathrm{A}_{I}$ and $\mathrm{D}_{X}$ of eqn. 2 utilize error signals from low-energy electron beam diagnostics $(1 \mathrm{GeV})$ and act on the laser beam. Additional independent diagnostics at both low and high $(50 \mathrm{GeV})$ energy are used to monitor the performance of the feedback loops. We choose to generate the error signals from beam diagnostics at low energy ${ }^{5}$ in order to minimize coupling between intensity, position, energy, and spotsize asymmetries due to beam loading, residual dispersion, and wakefield effects in the accelerator. Three factors enter into the requirements for the performance of the active feedback loops: the E158 tolerance, the beam jitter (which affects how long it takes to measure the parameter to the level of E158's tolerance), and how well we can initially null the asymmetry or difference.

We believe the main source of the intensity asymmetry is residual linear polarization due to the antisymmetric phase shifts discussed in the previous section. In principle, we know how to correct this asymmetry: measure the intensity asymmetry and adjust $\Delta_{1}$ and $\Delta_{2}$ accordingly. However, because the intensity jitter is $\sim 0.5 \%$ $1 . \%$, there will not be enough statistics $\left(\sim 6 \cdot 10^{8}\right.$ pulses $)$ in the experiment to ensure that the final asymmetry meets E158's requirement of an integrated intensity asymmetry below $2 \cdot 10^{-7}$. Thus, we not only require that the intensity feedback close to below $2 \cdot 10^{-7}$ but that it do so an order of magnitude faster than with statistical scaling. We measure the intensity asymmetry for a number of pulses (a "minirun" typically 800 pulses in length). If we have properly set the CP and PS cell voltages, the measured intensity asymmetry will be consistent with zero at the level of the statistics allowed by the length of the minirun. However, by applying a correction signal on the subsequent minirun that cancels the measured asymmetry, we cancel out the statistical fluctuations (up to the level of the measurement resolution, which at $3 \cdot 10^{-5}$ is much smaller than the $0.5-1.5 \%$ beam jitter). Ideally, for $\mathrm{N}$ miniruns, only the final minirun will contribute an asymmetry; the rest will have been arranged to cancel out. To the degree that this is true the asymmetry scales as $1 / \mathrm{N}$ rather than $1 / \operatorname{sqrt}(\mathrm{N})$. This $1 / \mathrm{N}$ scaling was demonstrated during T-437. ${ }^{5}$ The intensity asymmetry feedback is driven by introducing a helicity-correlated phase shift on the laser beam with the IA Pockels cell, which the Cleanup Polarizer transforms into the desired intensity asymmetry correction. The attenuation curve for the 
IA cell goes as $\cos ^{2} \phi$, where $\phi$ is the induced phase shift. Because the slope at $\phi=0$ is zero, we choose to run the IA cell nominally at $99 \%$ transmission in both states (approximately $475 \mathrm{~V}$ ) and to apply a correction by increasing the attenuation on the appropriate state in order to minimize the helicity-correlated voltage swing. The IA cell can be driven to $750 \mathrm{~V}$, yielding a range of asymmetry correction of approximately $\pm 1.0 \%$. The goal is to keep the intensity asymmetry correction below $10^{-4}$ over short time scales (5-10 minutes), and to average much closer to zero over the length of the experiment by using a "double feedback" in which we periodically adjust the offset phases $\Delta_{1}$ and $\Delta_{2}$ to minimize the correction applied by the IA cell.

E158 requires that the position differences be below $10 \mathrm{~nm}$ averaged over several months of data taking. The position jitter is typically on the order of 50-100 $\mu \mathrm{m}$ per pulse, so it should be possible to measure the position difference to a few $\mathrm{nm}$ without any assistance from the better-than-statistical scaling that an active feedback can provide. However, unlike the intensity asymmetry, where we believe we understand how to reliably null the asymmetry at its source, we don't understand how to reliably make the position differences consistent with zero before turning on the feedback. The helicity-correlated position differences are typically several microns at the cathode when no effort is made to control them; with various optimizations (choice of Pockels cells, translation of Pockels cells, and choice of operating voltages) we have achieved position differences as small as $1 \mu \mathrm{m}$. We also observe approximately an order of magnitude reduction in position differences between the cathode and the E158 target two miles downstream due to a combination of a drop in spot size (from a $5 \mathrm{~mm}$ sigma at the cathode to $1 \mathrm{~mm}$ at the target) and emittance growth through the accelerator. Thus, the goal is to achieve 1-2 orders of magnitude of suppression of position differences from an active feedback system. The second mirror on the Helicity Control Bench is mounted on a piezo stack that can be pulsed in a helicity-correlated manner. This mirror changes the angle of the laser beam through the remainder of the optical system and can produce helicity-correlated displacements on the cathode of 50-60 $\mu \mathrm{m}$, comparable to the beam position jitter. This "piezomirror" is the control device for the helicity-correlated position feedback system. The piezomirror is a standard 1" diameter mirror attached to a Physik Instrumente model S-311.10 ${ }^{17}$ piezoelectric mount. This unit has three piezoelectric stacks that can be pulsed individually up to $100 \mathrm{~V}$. The independent operation of the three stacks gives the freedom to translate the face of the mount up to $6 \mu \mathrm{m}$, or to tilt in an arbitrary direction by up to $600 \mu \mathrm{rad}$. Depending on the tune of the front end of the accelerator, the dynamic range of the feedback at the $1 \mathrm{GeV}$ beam diagnostics is typically 5-20 $\mu \mathrm{m}$. We choose a feedback minirun length such that the statistical error in the position difference measurement is approximately $10 \%$ of the dynamic range. This typically requires $1-4 \cdot 10^{4}$ pulses. T- 437 demonstrated that the position feedback converged with $1 / \mathrm{N}$ scaling at low energy, though during the E158 engineering runs we were unable to demonstrate this scaling using beam diagnostics at high energy.

Immediately downstream of the PS cell is a device called the Asymmetry Inverter. The Asymmetry Inverter is a translation stage carrying two sets of lenses: a doublet that provides a magnification of -2.25 and a quadruplet that provides a magnification of +2.25 . Both sets have an effective lever arm close to zero. Switching between the two states of the Asymmetry Inverter should invert both the spatial distribution of the laser beam's intensity profile and its angle leaving the CP and PS Pockels cells. In principle, running for equal amounts of time in each mode should yield a cancellation of any helicity-correlated position and angle effects arising from the polarization optics. Effects arising from downstream optics, however, would be uncorrected. A test of this cancellation during T-437 yielded encouraging results, as shown in Table 2, where for Y position differences, for which the applied correction was large, a significant degree of cancellation is seen.

Table 2. Size of corrections applied by helicity-correlated position feedback for alternate states of the Asymmetry Inverter. Reprinted from P. Mastromarino et al. ${ }^{5}$

\begin{tabular}{|l|l|l|l|}
\hline Fdbk Correction Induced & -2I State & $+2 \mathrm{I}$ State & Average \\
\hline X Position Difference & $262 \pm 48 \mathrm{~nm}$ & $-39 \pm 71 \mathrm{~nm}$ & $168 \pm 40 \mathrm{~nm}$ \\
\hline Y Position Difference & $-862 \pm 109 \mathrm{~nm}$ & $1157 \pm 130 \mathrm{~nm}$ & $-29 \pm 84 \mathrm{~nm}$ \\
\hline
\end{tabular}




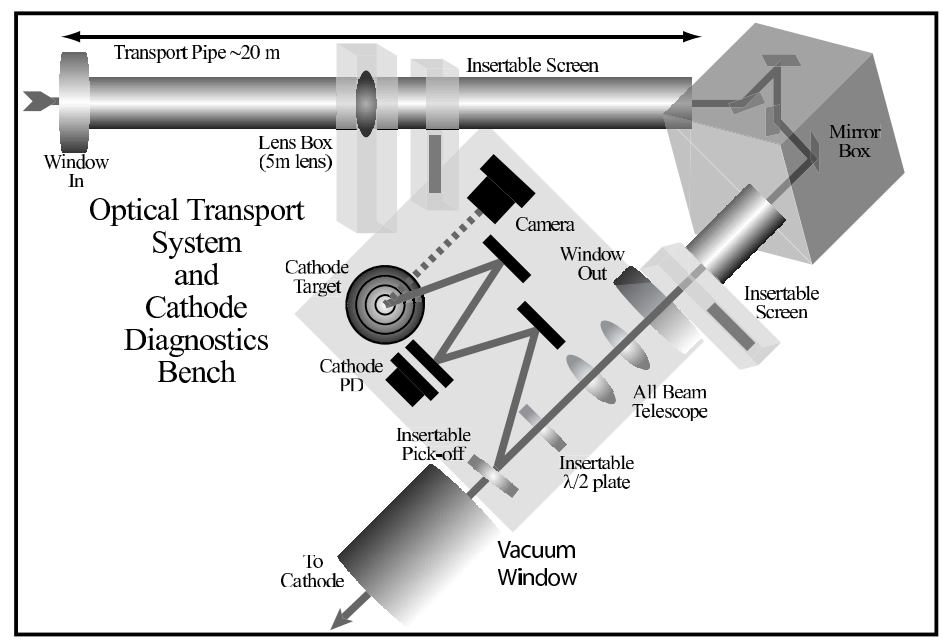

Figure 8: The optical transport system and the cathode diagnostics bench (not to scale).

After the Asymmetry Inverter are two remotely insertable optics mounts, the first containing a half-wave plate and the second containing the Helicity Filter that is used for setting the CP and PS cell voltages. The half-wave plate provides a slow reversal of the sign of the physics asymmetry, while leaving untouched certain classes of helicity-correlated systematics. In particular, helicity-correlated electronic cross-talk should not be affected by the insertion of a piece of glass into the beam, so in averaging equal amounts of half-wave plate "in" and "out" data, it should be cancelled out. Other non-polarization-related helicity-correlated systematics should behave similarly, but the behavior of systematics arising from imperfections in the circular polarization of the laser beam depends on the orientation of the half-wave plate in a complicated way, so it is not straightforward to gain a cancellation. There is a second half-wave plate on the Cathode Laser Diagnostics Bench for the same purpose, and during T-437 it did provide some cancellation of systematics, approximately a factor of three reduction in $\mathrm{Y}$ position differences and an order of magnitude in $\mathrm{X}$ position differences. ${ }^{5}$ For the physics run of the experiment, the quality of cancellation provided by each half-wave plate will be studied, and the one that provides a better cancellation will be used. We will likely flip the state of the half-wave plate every few hours.

Finally, we designed the laser transport system to image the Pockels cells onto the cathode. Studies have indicated that the Pockels cells can act as helicity-correlated lenses. By imaging the CP cell onto the cathode, we minimize the contribution of any helicity-correlated steering from the CP cell. In practice, it is the PS cell that is imaged, leaving an effective lever arm from the CP cell to the cathode of approximately $15 \mathrm{~cm}$. This is a significant reduction from the $\sim 25 \mathrm{~m}$ actual distance between the $\mathrm{CP}$ cell and the cathode. The telescope on the Cathode Diagnostics Bench fixes the location of the image point. That telescope is used to set the beam size on the cathode, and the location of the image point on the Helicity Control bench is very sensitive to its setting. We determine the location of the image point by placing a wire mesh screen in the beam and moving it along the beam axis while looking at the quality of the image on the Cathode Target (described below). The image point is observed to be the same for both states of the Asymmetry Inverter.

\section{OPTICAL TRANSPORT SYSTEM AND CATHODE LASER DIAGNOSTICS}

Fig. 8 shows the Optical Transport System (OTS) and the Cathode Laser Diagnostics bench. A $5 \mathrm{~m}$ focallength relay lens on a remotely controlled $x-y$ stage is located at the midway point of the OTS. A Mirror Box containing four helicity-compensated mirrors brings the beam onto the Cathode Laser Diagnostics Bench. Its mirrors are mounted in two helicity-compensated pairs, for which the bounces within each pair interchange 's' and 'p' polarizations. Care was taken to make certain that the mirrors all came from the same coating run. The OTS also contains two insertable screens for checking the beam position, the first about one meter downstream of the lens box and the second between the Mirror Box and the Cathode Laser Diagnostics Bench. The Cathode Laser Diagnostics Bench is located immediately before the Polarized Gun. The laser beam takes a straight path 
across the bench from the OTS exit window to the photocathode. The telescope is used to set the final size and position of the beam on the cathode. The telescope's upstream lens is mounted on an X-Y-Z translation stage and its downstream lens is fixed. A second remotely insertable half-wave plate can be used for a slow helicity reversal in the same manner as the one on the Helicity Control Bench. The remotely insertable $50 \%$ mirror immediately before the Cathode Laser Diagnostics Bench exit redirects the beam into a diagnostic line that has the same length as the distance between the pick-off and the photocathode. The Cathode Target provides an image of the beam as it appears on the actual photocathode and is an extremely useful diagnostic, in particular for understanding the imaging of the beam from the CP cell onto the cathode and for measuring the cathode's quantum efficiency dependence on position. Behind one of the mirrors in the diagnostic line is a photodiode that is used for checking the laser power reaching the cathode and for remotely setting the CP and PS voltages using the Helicity Filter.

\section{CONCLUSION}

We have described a laser system suitable for use in a polarized electron source for high-energy physics applications. The laser is a stable, high-power, high-repetition rate, low maintenance flashlamp-pumped Ti:sapphire. Polarized electrons of either helicity can be generated by flipping the helicity of the laser beam. The system has been optimized to minimize helicity correlations in the polarized electron beam. Active feedbacks on the helicity-correlated intensity asymmetry and position differences have been implemented. The insertable halfwave plates which flip the sign of the physics asymmetry and the Asymmetry Inverter which flips the sensitivity to systematics arising from the polarization Pockels cells should provide some degree of slow cancellation of systematics. Imaging of the polarization Pockels cells onto the photocathode also serves to help suppress systematics. This upgraded system will see its first use with the running of E158 in 2002/03 and will continue to be used for a variety of polarized beam experiments thereafter.

\section{REFERENCES}

1. C. Y. Prescott et al., "Further measurements of parity nonconservation in inelastic electron scattering," Phys. Lett. B 84, p. 524, 1979.

2. E. W. Hughes and R. Voss Ann. Rev. Nucl. Part. Sci. 49, pp. 303-339, 1999. See references contained therein.

3. P. C. Rowson, D. Su, and S. Willocq Ann. Rev. Nucl. Part. Sci. 51, 2001. See references contained therein.

4. R. Alley et al., "The Stanford Linear Accelerator polarized electron source," Nucl. Instrum. Meth. A365, pp. 1-27, 1995.

5. P. A. Mastromarino et al., "Helicity-correlated systematics for SLAC experiment E158," SLAC-PUB-9071.

6. K. H. Witte, "A reliable low maintenance flashlamp pumped ti:sapphire laser operating at 120-pps," Presented at Lasers '93, Lake Tahoe, NV, Dec 6-9, 1993.

7. Big Sky Laser Technologies, Bozeman, MT, USA.

8. Model L8061E, T J Sales Associates Inc., ILC Technology Inc., Denville, NJ, USA.

9. Union Carbide Crystal Products, Washougal, WA, USA.

10. KTF-2 fluorescent converter, Kigre, Inc., Hilton Head, SC, USA.

11. Directed Energy, Inc., Fort Collins, CO, USA.

12. Gentec Electro-Optics, Quebec, QC, Canada.

13. P. Horowitz and W. Hill, The Art of Electronics, Cambridge University Press, Cambridge, 1980. We implemented the pseudo-random helicity sequence according to the method described on pp. 437-442.

14. G. D. Cates et al., "The Bates polarized electron source," Nucl. Instrum. Meth. A278, pp. 293-317, 1989.

15. R. A. Mair et al., "Anisotropies in strain and quantum efficiency of strained GaAs grown on GaAsP," Phys. Lett. A212, pp. 231-236, 1996.

16. Cleveland Crystals, Inc., 676 Alpha Dr., Highland Hts., OH 44143, USA.

17. Physik Instrumente GmbH and Co, Auf der Roemerstrasse D-76228 Karlsruhe/Palmbach, Germany. 